\title{
Asymptotic expansion of radial solutions for supercritical biharmonic equations
}

\author{
Paschalis Karageorgis
}

\begin{abstract}
Consider the positive, radial solutions of the nonlinear biharmonic equation $\Delta^{2} \varphi=\varphi^{p}$. There is a critical power $p_{c}$ such that solutions are linearly stable if and only if $p \geq p_{c}$. We obtain their asymptotic expansion at infinity in the case that $p \geq p_{c}$.
\end{abstract}

Mathematics Subject Classification (2000). 35B40, 35J30.

\section{Introduction}

We study the positive, radial solutions of the nonlinear biharmonic equation

$$
\Delta^{2} \varphi(x)=\varphi(x)^{p}, \quad x \in \mathbb{R}^{n} .
$$

Such solutions are known to exist when $n>4$ and $p \geq \frac{n+4}{n-4}$, but they fail to exist, otherwise. Our goal in this paper is to derive their asymptotic expansion as $|x| \rightarrow \infty$ and thus obtain an analogue of a well-known result [8] for the second-order equation

$$
-\Delta \varphi(x)=\varphi(x)^{p}, \quad x \in \mathbb{R}^{n} .
$$

We remark that the qualitative properties of solutions to (1.1) resemble those of solutions to (1.2), however the methods used to establish them are quite different.

First, let us summarize the known results for the second-order equation (1.2). If $n \leq 2$ or $1<p<\frac{n+2}{n-2}$, then no positive solutions exist; and if $p=\frac{n+2}{n-2}$, then all positive solutions are radial up to a translation and also explicit [1,2]. If $p>\frac{n+2}{n-2}$, finally, the positive radial solutions form a one-parameter family $\left\{\varphi_{\alpha}\right\}_{\alpha>0}$, see $[5,11]$. When it comes to the behavior of the solutions $\varphi_{\alpha}$, a crucial role is played by a singular solution of the form

$$
\Phi(x)=a_{0}(n, p) \cdot|x|^{-\frac{2}{p-1}} .
$$

As $|x| \rightarrow \infty$, that is, each $\varphi_{\alpha}$ behaves like the singular solution $\Phi$. 
There is also a critical value $p_{c}$ associated with the second-order equation (1.2). This is defined by taking $\left(\frac{n+2}{n-2}, p_{c}\right)$ to be the maximal interval on which

$$
p \cdot Q_{2}\left(\frac{2}{p-1}\right)>Q_{2}\left(\frac{n-2}{2}\right), \quad Q_{2}(\alpha) \equiv|x|^{\alpha+2}(-\Delta)|x|^{-\alpha} .
$$

It is easy to check that $p_{c}=\infty$ if $n \leq 10$ and that $p_{c}<\infty$, otherwise. In the subcritical case $\frac{n+2}{n-2}<p<p_{c}$, each radial solution $\varphi_{\alpha}$ oscillates around the singular solution (1.3) and the graphs of any two radial solutions intersect one another [13]. In the supercritical case $p \geq p_{c}$, on the other hand, the graphs of distinct solutions $\varphi_{\alpha}$ do not intersect one another and they do not intersect the graph of the singular solution, either [13].

Let us now turn to the fourth-order equation (1.1). Although the known results are very similar to those listed above, their proofs are generally quite different. In this case, positive solutions fail to exist if $n \leq 4$ or $1<p<\frac{n+4}{n-4}$, while they are explicit and radial up to a translation, if $p=\frac{n+4}{n-4}$, see $[3,12,14]$. And if $p>\frac{n+4}{n-4}$, there is a one-parameter family of radial solutions $\varphi_{\alpha}$ which behave asymptotically like a singular solution of the form

$$
\Phi(x)=a_{0}(n, p) \cdot|x|^{-\frac{4}{p-1}},
$$

see [7]. The associated critical value arose in [7] and it is defined by taking $\left(\frac{n+4}{n-4}, p_{c}\right)$ to be the maximal interval on which

$$
p \cdot Q_{4}\left(\frac{4}{p-1}\right)>Q_{4}\left(\frac{n-4}{2}\right), \quad Q_{4}(\alpha) \equiv|x|^{\alpha+4} \Delta^{2}|x|^{-\alpha} .
$$

Moreover, $p_{c}<\infty$ if and only if $n \geq 13$, while the graphs of radial solutions intersect one another in the subcritical case $[6,9]$ but not in the supercritical case $[9,10]$.

There are also results that are well-known in the second-order case (1.2) but remain open in the fourth-order case (1.1). Those include the precise asymptotic expansion of the radial solutions in the supercritical case $p \geq p_{c}$. Expansions for the second-order case go back to $[8,11]$ and they provided a key ingredient for studying the stability of steady states for the nonlinear heat equation $u_{t}-\Delta u=u^{p}$ in the supercritical case [8]. Our goal in this paper is to establish an analogous expansion for the fourth-order problem.

Theorem 1.1. Suppose $n \geq 13$ and $p \geq p_{c}$. Let $\varphi$ be a positive, radial solution of (1.1) and let $\lambda_{1}<\lambda_{2} \leq \lambda_{3}<0<\lambda_{4}$ be the eigenvalues of the associated linearized equation, see Lemma 3.1. Then there exists a finite sequence $p_{c}=p_{1}<p_{2}<\cdots<p_{N}$ such that $\lambda_{2} \leq k \lambda_{3}$ if and only if $p \geq p_{k}$. Moreover, $\varphi$ has the following asymptotic expansion as $r \rightarrow \infty$.

(a) If $p_{k}<p<p_{k+1}$, with the convention that $p_{N+1}=\infty$, then

$$
r^{\frac{4}{p-1}} \varphi(r)=a_{0}+\sum_{j=1}^{k} a_{j} r^{j \lambda_{3}}+b_{1} r^{\lambda_{2}}+a_{k+1} r^{(k+1) \lambda_{3}}+O\left(r^{\lambda_{2}+\lambda_{3}}\right) .
$$


(b) If $p=p_{k}$ for some $k \geq 2$, then $k \lambda_{3}=\lambda_{2}$ and $r^{\frac{4}{p-1}} \varphi(r)=a_{0}+\sum_{j=1}^{k-1} a_{j} r^{j \lambda_{3}}+r^{k \lambda_{3}}\left(b_{1} \log r+a_{k}\right)+O\left(r^{\lambda_{2}+\lambda_{3}} \log r\right)$.

(c) If $p=p_{c}$, finally, then $\lambda_{2}=\lambda_{3}$ and $r^{\frac{4}{p-1}} \varphi(r)=a_{0}+r^{\lambda_{3}}\left(b_{1} \log r+a_{1}\right)+b_{2} r^{2 \lambda_{3}}(\log r)^{2}+O\left(r^{2 \lambda_{3}} \log r\right)$.

Preliminary versions of these expansions appeared in $[9,15]$. The expansion in [15] only lists two terms, but its proof is quite different from ours and contains some nice ideas. The expansion in [9] lists three terms, but it is not rigorously proven and not entirely correct in the critical case $p=p_{c}$.

The proof of Theorem 1.1 is given in Sect. 2; it heavily relies on the fact that $r^{\frac{4}{p-1}} \varphi(r)$ is increasing, as first observed by the author [10]. Although a similar statement holds in the second-order case [4], the corresponding proofs $[8,11]$ do not use that statement. Finally, Sect. 3 collects some basic facts about the quartic polynomial (1.6) and the eigenvalues of the associated linearized equation; we use these facts in the proof of our main result.

\section{Asymptotic expansion at infinity}

In this section, we give the proof of Theorem 1.1 regarding the asymptotic expansion of the positive, radial solutions of (1.1). First, we use an EmdenFowler transformation to transform (1.1) into an ODE whose linear part has constant coefficients. Then, we analyze this ODE using some key results of Gazzola-Grunau [7] and the author [10].

Lemma 2.1. Let $p>1$ and $m=\frac{4}{p-1}$. If $\varphi$ is a positive, radial solution of (1.1), then

$$
W(s)=e^{m s} \varphi\left(e^{s}\right)=r^{m} \varphi(r), \quad s=\log r=\log |x|
$$

is a solution of the ordinary differential equation

$$
Q_{4}\left(m-\partial_{s}\right) W(s)=W(s)^{p},
$$

where $Q_{4}$ is the quartic polynomial defined by

$$
Q_{4}(\alpha)=|x|^{\alpha+4} \Delta^{2}|x|^{-\alpha}=\alpha(\alpha+2)(\alpha+2-n)(\alpha+4-n) .
$$

Proof. Since $\partial_{r}=e^{-s} \partial_{s}$, a short computation allows us to write the radial Laplacian as

$$
\Delta=\partial_{r}^{2}+(n-1) r^{-1} \partial_{r}=e^{-2 s}\left(n-2+\partial_{s}\right) \partial_{s} .
$$

Using the operator identity $\partial_{s} e^{-k s}=e^{-k s}\left(\partial_{s}-k\right)$, one can then easily check that

$$
\Delta^{2} e^{-m s}=e^{-4 s-m s} Q_{4}\left(m-\partial_{s}\right)=e^{-m p s} Q_{4}\left(m-\partial_{s}\right) .
$$

This also implies that $Q_{4}\left(m-\partial_{s}\right) W(s)=e^{m p s} \Delta^{2} \varphi\left(e^{s}\right)=W(s)^{p}$, as needed. 
Lemma 2.2. Suppose that $n>4$ and $p>\frac{n+4}{n-4}$. Then the positive, radial solutions of (1.1) form a one-parameter family $\left\{\varphi_{\alpha}\right\}_{\alpha>0}$, where each $\varphi_{\alpha}$ satisfies $\varphi_{\alpha}(0)=\alpha$ and

$$
\lim _{r \rightarrow \infty} r^{m} \varphi_{\alpha}(r)=Q_{4}(m)^{\frac{1}{p-1}}
$$

with $m=\frac{4}{p-1}$ and $Q_{4}$ as in (2.3). If we also assume that $p \geq p_{c}$, then

$$
Y=r^{m} \varphi_{\alpha}(r)-Q_{4}(m)^{\frac{1}{p-1}}
$$

is strictly increasing for all $r>0$, hence also negative for all $r>0$.

Proof. See Theorem 1 in [7] for the existence part, Theorem 3 in [7] for a proof of (2.4) and equation (4.7) in [10] for the monotonicity of $Y$ in the supercritical case.

To understand the behavior of the function $Y$ in (2.5), we use Lemma 2.1 to get

$$
\left[Q_{4}\left(m-\partial_{s}\right)-p Q_{4}(m)\right] Y(s)=(Y+L)^{p}-L^{p}-p L^{p-1} Y \equiv g(Y),
$$

where $s=\log r$ and $L=Q_{4}(m)^{\frac{1}{p-1}}$. Note that the linearized equation is given by the left hand side. As we shall show in Lemma 3.1, the associated eigenvalues are all real in the supercritical case $p \geq p_{c}$ and they also satisfy

$$
\lambda_{1}<\lambda_{2} \leq \lambda_{3}<0<\lambda_{4} .
$$

The presence of a positive eigenvalue is likely to complicate matters because we are seeking an expansion as $s \rightarrow \infty$. We thus isolate this eigenvalue and we factor (2.6) as

$$
\left(\partial_{s}-\lambda_{1}\right)\left(\partial_{s}-\lambda_{2}\right)\left(\partial_{s}-\lambda_{3}\right) Z(s)=g(Y), \quad Z(s) \equiv Y^{\prime}(s)-\lambda_{4} Y(s) .
$$

Since $Y(s)$ is negative and increasing by Lemma 2.2, we actually have

$$
Z(s)=\left|Y^{\prime}(s)\right|+\left|\lambda_{4} Y(s)\right|
$$

and we can use Gronwall-type estimates to control $Z(s)$; this is where Lemma 2.2 becomes crucial, as it ensures that $Z(s) \geq 0$. Once we have some precise estimate for $Z(s)$, we can simply integrate to get an estimate for $Y(s)$, and we can then repeatedly use the following lemma to obtain refined expansions for both $Z(s)$ and $Y(s)$.

Lemma 2.3. Suppose $n \geq 13$ and $p \geq p_{c}$. Let $Z(s)$ be as in (2.8). Given any $s_{0} \in \mathbb{R}$ then, there exist some constants $\alpha_{i}, \beta_{i}$ such that

$$
Z(s)=\sum_{i=1}^{3} \alpha_{i} e^{\lambda_{i} s}+\beta_{i} \int_{s_{0}}^{s} e^{\lambda_{i}(s-\tau)} \cdot g(Y(\tau)) d \tau
$$

in the supercritical case $p>p_{c}$ and

$$
\begin{aligned}
Z(s)= & \sum_{i=1}^{2} \alpha_{i} e^{\lambda_{i} s}+\beta_{i} \int_{s_{0}}^{s} e^{\lambda_{i}(s-\tau)} \cdot g(Y(\tau)) d \tau \\
& +\alpha_{3} s e^{\lambda_{3} s}+\beta_{3} \int_{s_{0}}^{s}(s-\tau) e^{\lambda_{3}(s-\tau)} \cdot g(Y(\tau)) d \tau
\end{aligned}
$$


in the critical case $p=p_{c}$. Moreover, each $\alpha_{i}$ depends on $s_{0}$ and the eigenvalues $\lambda_{1}, \lambda_{2}, \lambda_{3}$ which appear in equation (2.8), whereas each $\beta_{i}$ depends solely on the eigenvalues.

Proof. We multiply the first equation in (2.8) by $e^{-\lambda_{1} s}$ and we integrate to get

$$
\left(\partial_{s}-\lambda_{2}\right)\left(\partial_{s}-\lambda_{3}\right) Z(s)=A_{1} e^{\lambda_{1} s}+\int_{s_{0}}^{s} e^{\lambda_{1}(s-\tau)} \cdot g(Y(\tau)) d \tau
$$

for some constant $A_{1}$. Repeating the same argument once again, we arrive at

$$
\left(\partial_{s}-\lambda_{3}\right) Z(s)=B_{1} e^{\lambda_{1} s}+B_{2} e^{\lambda_{2} s}+\int_{s_{0}}^{s} \int_{s_{0}}^{\rho} e^{\lambda_{2}(s-\rho)} e^{\lambda_{1}(\rho-\tau)} \cdot g(Y(\tau)) d \tau d \rho
$$

because $\lambda_{1}<\lambda_{2}$. Once we now switch the order of integration, we get

$$
\begin{aligned}
\left(\partial_{s}-\lambda_{3}\right) Z(s) & =B_{1} e^{\lambda_{1} s}+B_{2} e^{\lambda_{2} s}+\int_{s_{0}}^{s} \int_{\tau}^{s} e^{\lambda_{2}(s-\rho)} e^{\lambda_{1}(\rho-\tau)} \cdot g(Y(\tau)) d \rho d \tau \\
& =B_{1} e^{\lambda_{1} s}+B_{2} e^{\lambda_{2} s}+\int_{s_{0}}^{s} \frac{e^{\lambda_{1}(s-\tau)}-e^{\lambda_{2}(s-\tau)}}{\lambda_{1}-\lambda_{2}} \cdot g(Y(\tau)) d \tau
\end{aligned}
$$

In the supercritical case, $\lambda_{2}<\lambda_{3}$ by Lemma 3.1, so we can repeat our approach once again to deduce (2.10). In the critical case, $\lambda_{2}=\lambda_{3}$ so our approach leads to $(2.11)$.

Lemma 2.4. Suppose $n \geq 13$ and $p \geq p_{c}$. Let $Z(s)$ be as in (2.8) and let $\delta>0$. Then

$$
Z(s)=O\left(e^{\lambda_{3} s+\delta s}\right) \quad \text { as } s \rightarrow \infty .
$$

Proof. Suppose first that $p>p_{c}$. Then $\lambda_{1}<\lambda_{2}<\lambda_{3}$ by Lemma 3.1 and so

$$
Z(s) \leq C_{1} e^{\lambda_{3} s}+C_{2} \int_{s_{0}}^{s} e^{\lambda_{3}(s-\tau)} \cdot|g(Y(\tau))| d \tau
$$

by (2.10). Now, Lemma 2.2 and our definition (2.6) ensure that

$$
\lim _{\tau \rightarrow \infty} \frac{g(Y(\tau))}{Y(\tau)}=g^{\prime}(0)=0
$$

Since $Z(s) \geq\left|\lambda_{4} Y(s)\right|$ by equation (2.9), this trivially implies

$$
|g(Y(\tau))| \leq \frac{\delta\left|\lambda_{4} Y(\tau)\right|}{C_{2}} \leq \frac{\delta Z(\tau)}{C_{2}}
$$

for all large enough $\tau$. Inserting this estimate in (2.13), we conclude that

$$
e^{-\lambda_{3} s} Z(s) \leq C_{1}+\delta \int_{s_{0}}^{s} e^{-\lambda_{3} \tau} Z(\tau) d \tau
$$

for all large enough $s_{0}, s$. Using Gronwall's inequality, we thus obtain (2.12).

Suppose now that $p=p_{c}$, in which case $\lambda_{1}<\lambda_{2}=\lambda_{3}$ by Lemma 3.1. Using the exact same approach as above with (2.11) instead of (2.10), we now get

$$
e^{-\lambda_{3} s} Z(s) \leq C_{1} s+\frac{\delta}{2} \int_{s_{0}}^{s} e^{-\lambda_{3} \tau} Z(\tau) d \tau+\frac{\delta^{2}}{2} \int_{s_{0}}^{s}(s-\tau) e^{-\lambda_{3} \tau} Z(\tau) d \tau
$$


for all large enough $s_{0}, s$ in analogy with (2.14). Letting $F(s)$ denote the rightmost integral, one can express the last equation in the form

$$
F^{\prime \prime}(s) \leq C_{1} s+\frac{\delta}{2} F^{\prime}(s)+\frac{\delta^{2}}{2} F(s), \quad F\left(s_{0}\right)=F^{\prime}\left(s_{0}\right)=0 .
$$

We note that $F, F^{\prime}$ are non-negative, while $G(s)=F^{\prime}(s)+\frac{\delta}{2} F(s)$ is such that

$$
G^{\prime}(s)-\delta G(s)=F^{\prime \prime}(s)-\frac{\delta}{2} F^{\prime}(s)-\frac{\delta^{2}}{2} F(s) \leq C_{1} s .
$$

Multiplying by $e^{-\delta s}$ and integrating, we now get

$$
F^{\prime}(s)+\frac{\delta}{2} F(s)=G(s) \leq C_{1} e^{\delta s} \int_{s_{0}}^{s} \tau e^{-\delta \tau} d \tau \leq C_{2} e^{\delta s} .
$$

Since $F, F^{\prime}$ are non-negative by above, we may thus recall (2.15) to conclude that

$$
e^{-\lambda_{3} s} Z(s)=F^{\prime \prime}(s) \leq C_{1} s+C_{3} e^{\delta s} .
$$

This trivially implies the desired (2.12) and also completes the proof.

Lemma 2.5. Under the assumptions of the previous lemma, one has

$$
Z(s)=\left\{\begin{array}{ll}
O\left(s e^{\lambda_{3} s}\right) & \text { if } p=p_{c} \\
O\left(e^{\lambda_{3} s}\right) & \text { if } p>p_{c}
\end{array}\right\} .
$$

Proof. Let us fix some $0<\delta<\left|\lambda_{3}\right| / 2$ and consider two cases.

Case 1. When $p>p_{c}$, we use equation (2.10) to get

$$
Z(s) \leq C_{1} e^{\lambda_{3} s}+C_{2} \int_{s_{0}}^{s} e^{\lambda_{3}(s-\tau)} \cdot|g(Y(\tau))| d \tau .
$$

According to (2.6) and (2.9), the rightmost factor in the integrand is bounded by

$$
|g(Y(\tau))| \leq C Y(\tau)^{2} \leq C Z(\tau)^{2}
$$

for all large enough $\tau$. Using this fact and the previous lemma, we find that

$$
e^{-\lambda_{3} s} Z(s) \leq C_{1}+C \int_{s_{0}}^{s} e^{\left(\lambda_{3}+2 \delta\right) \tau} d \tau
$$

for all large enough $s_{0}, s$. Since $2 \delta<-\lambda_{3}$, the result now follows.

Case 2. When $p=p_{c}$, we use (2.11) instead of (2.10). Proceeding as above, one gets

$$
e^{-\lambda_{3} s} Z(s) \leq C_{3} s+C_{3} \int_{s_{0}}^{s} e^{\left(\lambda_{3}+2 \delta\right) \tau} d \tau+C_{3} \int_{s_{0}}^{s}(s-\tau) e^{\left(\lambda_{3}+2 \delta\right) \tau} d \tau
$$

instead of (2.17). Since $2 \delta<-\lambda_{3}$, the result follows as before.

Corollary 2.6. Under the assumptions of Lemma 2.4, one also has

$$
Y(s)=\left\{\begin{array}{ll}
O\left(s e^{\lambda_{3} s}\right) & \text { if } p=p_{c} \\
O\left(e^{\lambda_{3} s}\right) & \text { if } p>p_{c}
\end{array}\right\} .
$$


Proof. Recall our definition (2.8) which reads

$$
\left[e^{-\lambda_{4} s} Y(s)\right]^{\prime}=e^{-\lambda_{4} s} Z(s)
$$

for some $\lambda_{4}>0$. Since $Y(s) \rightarrow 0$ as $s \rightarrow \infty$ by (2.4), we may then integrate to get

$$
Y(s)=-\int_{s}^{\infty} e^{\lambda_{4}(s-\tau)} Z(\tau) d \tau .
$$

Using the expansion (2.16) for $Z(\tau)$, we obtain the expansion (2.18) for $Y(s)$.

Remark 2.7. In what follows, we shall frequently use the fact that

$$
\int_{s_{0}}^{s} O\left(e^{\mu \tau}\right) d \tau=\int_{s_{0}}^{\infty} O\left(e^{\mu \tau}\right) d \tau-\int_{s}^{\infty} O\left(e^{\mu \tau}\right) d \tau=C_{1}+O\left(e^{\mu s}\right)
$$

whenever $\mu<0$ as well as the analogous statement

$$
\int_{s_{0}}^{s} O\left(e^{\mu \tau}\right) d \tau=O\left(e^{\mu s}\right)
$$

whenever $\mu>0$. Moreover, similar statements hold with $e^{\mu s}$ replaced by $s e^{\mu s}$.

Proof of Theorem 1.1. Our assertions about the eigenvalues $\lambda_{i}$ and the critical values $p_{i}$ are basically facts about the quartic polynomial (2.3), so we establish them separately in Sect. 3, see Lemmas 3.1 and 3.4, respectively. In what follows, we may thus focus solely on the asymptotic expansions stated in the theorem.

For part (a), we assume $p_{k}<p<p_{k+1}$. In this case, we shall prove the expansions

$$
Y(s)=\sum_{j=1}^{l} a_{j} e^{j \lambda_{3} s}+O\left(e^{(l+1) \lambda_{3} s}\right), \quad 0 \leq l \leq k-1
$$

with the sum interpreted as zero when $l=0$, as well as the refined expansion

$$
Y(s)=\sum_{j=1}^{k} a_{j} e^{j \lambda_{3} s}+b_{1} e^{\lambda_{2} s}+O\left(e^{(k+1) \lambda_{3} s}\right) .
$$

Note that the former holds when $l=0$ by Corollary 2.6. To establish them both at the same time, we will show that (2.20) with $l<k-1$ implies (2.20) with $l+1$ and that (2.20) with $l=k-1$ implies (2.21).

Suppose then that (2.20) holds for some $0 \leq l \leq k-1$. In view of (2.6), the Taylor series expansion of $g$ near $Y=0$ has the form

$$
g(Y)=\sum_{j=2}^{L} d_{j} Y^{j}+O\left(Y^{L+1}\right)
$$


where $L$ is an arbitrary positive integer. We take $L=l+1$ and use (2.20) to get

$$
g(Y(s))=\sum_{j=2}^{l+1} c_{j} e^{j \lambda_{3} s}+O\left(e^{(l+2) \lambda_{3} s}\right) .
$$

The corresponding expression for $Z(s)$ provided by Lemma 2.3 is

$$
Z(s)=\sum_{i=1}^{3} \alpha_{i} e^{\lambda_{i} s}+\beta_{i} \int_{s_{0}}^{s} e^{\lambda_{i}(s-\tau)} \cdot g(Y(\tau)) d \tau
$$

and we may combine the last two equations to arrive at

$$
Z(s)=\sum_{i=1}^{3} \alpha_{i} e^{\lambda_{i} s}+\beta_{i} e^{\lambda_{i} s} \int_{s_{0}}^{s}\left[\sum_{j=2}^{l+1} c_{j} e^{\left(j \lambda_{3}-\lambda_{i}\right) \tau}+O\left(e^{(l+2) \lambda_{3} \tau-\lambda_{i} \tau}\right)\right] d \tau .
$$

Since $p_{k}<p<p_{k+1}$ by assumption, Lemmas 3.1 and 3.4 ensure that

$$
\lambda_{1}<\lambda_{2}+\lambda_{3}<(k+1) \lambda_{3}<\lambda_{2}<k \lambda_{3}<0 .
$$

In particular, $j \lambda_{3}-\lambda_{1}$ is positive for each $j \leq l+2 \leq k+1$, whereas $j \lambda_{3}-\lambda_{2}$ is positive when $j \leq k$ and negative when $j \geq k+1$. Thus, (2.24) leads to the expansion

$$
Z(s)=\sum_{j=1}^{l+1} a_{j} e^{j \lambda_{3} s}+O\left(e^{(l+2) \lambda_{3} s}\right)
$$

if $l \leq k-2$, but it leads to the expansion

$$
Z(s)=\sum_{j=1}^{k} a_{j} e^{j \lambda_{3} s}+b_{1} e^{\lambda_{2} s}+O\left(e^{(k+1) \lambda_{3} s}\right),
$$

if $l=k-1$. In either case, a similar expansion is easily seen to hold for

$$
Y(s)=-\int_{s}^{\infty} e^{\lambda_{4}(s-\tau)} Z(\tau) d \tau
$$

by (2.19). This shows that (2.20) with $l<k-1$ implies (2.20) with $l+1$ and that (2.20) with $l=k-1$ implies (2.21). In particular, (2.21) follows by induction.

We now repeat this argument to refine (2.21) even further. As before, we insert (2.21) in (2.22) with $L=k+1$ and we end up with

$$
g(Y(s))=\sum_{j=2}^{k+1} c_{j} e^{j \lambda_{3} s}+O\left(e^{\left(\lambda_{2}+\lambda_{3}\right) s}\right) .
$$

Inserting the last equation in (2.23) and recalling (2.25), we deduce that

$$
Z(s)=\sum_{j=1}^{k} a_{j} e^{j \lambda_{3} s}+b_{1} e^{\lambda_{2} s}+a_{k+1} e^{(k+1) \lambda_{3} s}+O\left(e^{\left(\lambda_{2}+\lambda_{3}\right) s}\right) .
$$


By (2.19), a similar expansion holds for $Y(s)$, so the expansion (1.7) for part (a) follows.

For part (b), we assume $p=p_{k}$ with $k \geq 2$. In this case, we shall similarly prove

$$
Y(s)=\sum_{j=1}^{l} a_{j} e^{j \lambda_{3} s}+O\left(e^{(l+1) \lambda_{3} s}\right), \quad 0 \leq l \leq k-2
$$

together with the refined expansion

$$
Y(s)=\sum_{j=1}^{k-1} a_{j} e^{j \lambda_{3} s}+O\left(s e^{k \lambda_{3} s}\right) .
$$

Note that the former holds when $l=0$ by Corollary 2.6. Proceeding as before, we assume (2.26) holds for some $0 \leq l \leq k-2$ and insert (2.26) in (2.22) with $L=l+1$. As $(2.26)$ is the same expansion that we had before, we still end up with (2.24), but we now have

$$
\lambda_{1}<\lambda_{2}+\lambda_{3}=(k+1) \lambda_{3}<\lambda_{2}=k \lambda_{3}<0
$$

instead of (2.25). Assuming $l<k-2$, equation (2.24) leads to (2.26) with $l+1$ exactly as before. If $l=k-2$, on the other hand, then it leads to (2.27).

We now repeat this argument to refine (2.27) even further. Inserting (2.27) in (2.22) with $L=k$, one finds that

$$
g(Y(s))=\sum_{j=2}^{k} c_{j} e^{j \lambda_{3} s}+O\left(s e^{(k+1) \lambda_{3} s}\right) .
$$

We combine this fact with (2.23) and recall (2.28) to get

$$
Z(s)=\sum_{j=1}^{k-1} a_{j} e^{j \lambda_{3} s}+b_{1} s e^{k \lambda_{3} s}+a_{k} e^{k \lambda_{3} s}+O\left(s e^{(k+1) \lambda_{3} s}\right) .
$$

Once again, this also implies the desired expansion (1.8) for part (b).

For part (c), finally, we assume $p=p_{1}$. In this case, (2.27) with $k=1$ is already known to hold by Corollary 2.6. To refine this expansion, we insert it in (2.22) with $L=1$ and proceed as before to find that

$$
Z(s)=b_{1} s e^{\lambda_{3} s}+a_{1} e^{\lambda_{3} s}+O\left(s^{2} e^{2 \lambda_{3} s}\right) .
$$

Using this and (2.22) with $L=2$, we may then repeat the same approach once more to end up with the expansion (1.9) which is stated in the theorem.

\section{Useful facts}

In this section, we gather some basic facts related to the quartic polynomial (2.3) in the supercritical case $p \geq p_{c}>\frac{n+4}{n-4}$, in which case

$$
\lambda_{*} \equiv \frac{4}{p-1}-\frac{n-4}{2}<0 \text {. }
$$


Lemma 3.1. Suppose $n \geq 13$ and $p \geq p_{c}$. If $m=\frac{4}{p-1}$ and $Q_{4}$ is the quartic (2.3), then

$$
\mathscr{P}(\lambda)=Q_{4}(m-\lambda)-p Q_{4}(m)
$$

has four real roots $\lambda_{1}<2 \lambda_{*}<\lambda_{2} \leq \lambda_{*} \leq \lambda_{3}<0<\lambda_{4}$ and those are such that

$$
\lambda_{1}+\lambda_{4}=\lambda_{2}+\lambda_{3}=2 \lambda_{*} \text {. }
$$

Moreover, a double root arises if and only if $p=p_{c}$, in which case $\lambda_{2}=\lambda_{3}=$ $\lambda_{*}$.

Proof. Noting that $Q_{4}$ is symmetric about $\frac{n-4}{2}$, we see that $\mathscr{P}$ is symmetric about

$$
\lambda_{*} \equiv m-\frac{n-4}{2}=\frac{4}{p-1}-\frac{n-4}{2}<0 .
$$

In addition, we have

$$
\lim _{\lambda \rightarrow \pm \infty} \mathscr{P}(\lambda)=+\infty, \quad \mathscr{P}\left(2 \lambda_{*}\right)=\mathscr{P}(0)=(1-p) \cdot Q_{4}(m)<0
$$

and we also have

$$
\mathscr{P}\left(\lambda_{*}\right)=Q_{4}\left(\frac{n-4}{2}\right)-p \cdot Q_{4}\left(\frac{4}{p-1}\right) \geq 0
$$

because $p \geq p_{c}$. This forces $\mathscr{P}(\lambda)$ to have at least one root in each of the intervals

$$
\left(-\infty, 2 \lambda_{*}\right), \quad\left(2 \lambda_{*}, \lambda_{*}\right], \quad\left[\lambda_{*}, 0\right), \quad(0, \infty) .
$$

If $\lambda_{*}$ happens to be a root, then it must be a double root by symmetry; this is only the case when equality holds in (3.5), namely when $p=p_{c}$. As for our assertion (3.3), this also follows by symmetry because $\lambda$ is a root of $\mathscr{P}$ if and only if $2 \lambda_{*}-\lambda$ is.

Lemma 3.2. Suppose $n \geq 13$ and $p \geq p_{c}$. Let $\lambda_{2} \leq \lambda_{3}<0$ be as in Lemma 3.1 and let $Q_{4}$ be the quartic polynomial (2.3). Given any integer $k \geq 1$, we then have

$$
\lambda_{2}>k \lambda_{3} \Longleftrightarrow \mathscr{R}_{k}(p)<0,
$$

where $\mathscr{R}_{k}(p)$ is the polynomial defined by

$$
\mathscr{R}_{k}(p)=(p-1)^{4}\left[Q_{4}\left(\frac{k-1}{k+1} \cdot \frac{4}{p-1}+\frac{n-4}{k+1}\right)-p Q_{4}\left(\frac{4}{p-1}\right)\right] .
$$

Proof. First of all, we use our identity (3.3) to find that

$$
\lambda_{2}>k \lambda_{3} \Longleftrightarrow 2 \lambda_{*}=\lambda_{2}+\lambda_{3}>(k+1) \lambda_{3},
$$

where $\lambda_{*}<0$ is defined by (3.1). According to Lemma 3.1, $\lambda_{3}$ is the unique root of

$$
\mathscr{P}(\lambda)=Q_{4}\left(\frac{4}{p-1}-\lambda\right)-p Q_{4}\left(\frac{4}{p-1}\right)
$$


that lies in the interval $\left[\lambda_{*}, 0\right)$. Since $k \geq 1$ by assumption, $\frac{2 \lambda_{*}}{k+1}$ also lies in that interval, while equations (3.4) and (3.5) give $\mathscr{P}\left(\lambda_{*}\right) \geq 0>\mathscr{P}(0)$. In particular, we have

$$
\lambda_{2}>k \lambda_{3} \Longleftrightarrow \frac{2 \lambda_{*}}{k+1}>\lambda_{3} \Longleftrightarrow \mathscr{P}\left(\frac{2 \lambda_{*}}{k+1}\right)<0,
$$

where $\lambda_{*}=\frac{4}{p-1}-\frac{n-4}{2}$ by definition (3.1), so the desired condition (3.6) follows.

Lemma 3.3. Suppose $n \geq 13$ and $p \geq p_{c}$. Let $k>1$ be an integer, let $\mathscr{R}_{k}(p)$ denote the polynomial (3.7) of the previous lemma, and let $L$ denote the limit

$$
L=\lim _{p \rightarrow \pm \infty} \frac{\mathscr{R}_{k}(p)}{p^{4}}=Q_{4}\left(\frac{n-4}{k+1}\right)-8(n-2)(n-4) .
$$

(a) If $L \leq 0$, then $\mathscr{R}_{k}(p)$ is negative on $\left[p_{c}, \infty\right)$.

(b) If $L>0$, then $\mathscr{R}_{k}(p)$ has a unique root $p_{k}>p_{c}$ and it is negative on $\left[p_{c}, p_{k}\right)$ but positive on $\left(p_{k}, \infty\right)$.

Proof. First, we show that $\mathscr{R}_{k}(p)$ has two roots in $\left(1, p_{c}\right)$ by showing that

$$
\mathscr{R}_{k}(1)<0, \quad \mathscr{R}_{k}\left(\frac{n}{n-4}\right)>0, \quad \mathscr{R}_{k}\left(p_{c}\right)<0 .
$$

Using our definition (2.3), one can easily check that

$$
\lim _{p \rightarrow 1}(p-1)^{4} \cdot Q_{4}\left(\frac{a}{p-1}\right)=a^{4}
$$

for any $a \in \mathbb{R}$ whatsoever; in view of our definition (3.7), this also implies

$$
\mathscr{R}_{k}(1)=4^{4}\left(\frac{k-1}{k+1}\right)^{4}-4^{4}<0 .
$$

Next, we note that $Q_{4}$ is positive on $(0, n-4)$ with $Q_{4}(n-4)=0$, hence

$$
\mathscr{R}_{k}\left(\frac{n}{n-4}\right)=\left(\frac{4}{n-4}\right)^{4} \cdot Q_{4}\left(\frac{k(n-4)}{k+1}\right)>0 .
$$

To show that $\mathscr{R}_{k}\left(p_{c}\right)<0$, finally, we combine Lemma 3.1 with (3.6). When $p=p_{c}$, the lemma gives $\lambda_{2}=\lambda_{3}<0$, hence $\lambda_{2}>k \lambda_{3}$ and so $\mathscr{R}_{k}\left(p_{c}\right)<0$ by (3.6).

This completes the proof of (3.9), which implies that $\mathscr{R}_{k}(p)$ has two roots in $\left(1, p_{c}\right)$. To find the location of the remaining roots, we shall now have to distinguish two cases.

Case 1. When $n \leq 2(k+1)$, there are two additional roots in $[-1,1)$ because

$$
\mathscr{R}_{k}(-1) \leq 0, \quad \mathscr{R}_{k}(-1 / 3)>0, \quad \mathscr{R}_{k}(1)<0 .
$$

Assuming this statement for the moment, $\mathscr{R}_{k}$ has no roots in $\left[p_{c}, \infty\right)$, so it must be negative there by (3.9), and thus the limit (3.8) is non-positive; in fact, the limit has to be negative, as it can only be zero when $\mathscr{R}_{k}$ is a cubic instead of a quartic. 
To finish the proof for this case, we now establish (3.11). First of all, we have

$$
\mathscr{R}_{k}(-1)=2^{4} \cdot Q_{4}\left(\frac{n}{k+1}-2\right) \leq 0
$$

because $Q_{4}$ is non-positive on $(-2,0]$. Since $\mathscr{R}_{k}(1)<0$ by $(3.9)$, it remains to show

$$
\begin{aligned}
(3 / 4)^{4} \cdot \mathscr{R}_{k}(-1 / 3) & =Q_{4}\left(\frac{n+2}{k+1}-3\right)+\frac{Q_{4}(-3)}{3} \\
& =Q_{4}\left(\frac{n}{k+1}-2-\frac{k-1}{k+1}\right)+n^{2}-1
\end{aligned}
$$

is positive. In particular, it suffices to show that

$$
Q_{4}(x)+n^{2}-1>0 \text { whenever }-3<x<0 .
$$

Since $Q_{4}$ is positive on $(-3,-2)$, we may assume $-2 \leq x<0$. Then

$$
x(x+2) \geq-1 \quad \Longrightarrow \quad Q_{4}(x) \geq-(x+2-n)(x+4-n)
$$

and the rightmost quadratic is increasing on $(-\infty, 0)$, so we easily get

$$
Q_{4}(x) \geq n(2-n)>1-n^{2} .
$$

Case 2. When $n>2(k+1)$, there is one additional root in $(-1,1)$ because

$$
\mathscr{R}_{k}(-1)>0, \quad \mathscr{R}_{k}(1)<0 .
$$

This follows easily by (3.9) and the fact that $Q_{4}$ is positive on $(0, n-4)$, which gives

$$
\mathscr{R}_{k}(-1)=2^{4} \cdot Q_{4}\left(\frac{n}{k+1}-2\right)>0
$$

for this case. In view of $(3.9)$, we now know that $\mathscr{R}_{k}$ has three roots in $\left(-1, p_{c}\right)$, being positive at the left endpoint and negative at the right endpoint. If the limit (3.8) is positive, then $\mathscr{R}_{k}$ is positive as $p \rightarrow \infty$, so the fourth root lies in $\left(p_{c}, \infty\right)$; if the limit is zero, then $\mathscr{R}_{k}$ is a cubic, so it has no other roots; and if the limit is negative, then $\mathscr{R}_{k}$ is negative as $p \rightarrow-\infty$, so the fourth root lies in $(-\infty,-1)$. The result now follows.

Lemma 3.4. Suppose $n \geq 13$ and $p \geq p_{c}$. Let $\lambda_{2} \leq \lambda_{3}<0$ be as in Lemma 3.1. Then there exists a finite sequence $p_{c}=p_{1}<p_{2}<\cdots<p_{N}$ such that $\lambda_{2}>(N+1) \lambda_{3}$ and

$$
\lambda_{2} \leq k \lambda_{3} \Longleftrightarrow p \geq p_{k}
$$

for each $1 \leq k \leq N$. Moreover, if $\lfloor x\rfloor$ denotes the greatest integer in $x$, then the length of this finite sequence is

$$
N=\left\{\begin{array}{l}
\left\lfloor\frac{n-10}{2}\right\rfloor \text { when } 13 \leq n \leq 19 \\
\left\lfloor\frac{n-9}{2}\right\rfloor \text { when } n \geq 20
\end{array}\right\} .
$$


Proof. According to the previous two lemmas, it suffices to show that the limit (3.8) is positive when $k \leq N$ but negative when $k \geq N+1$. Let us thus focus on the quartic

$$
\mathscr{F}(k) \equiv \frac{2(k+1)^{4}}{n-4} \cdot\left[Q_{4}\left(\frac{n-4}{k+1}\right)-8(n-2)(n-4)\right]
$$

which is merely the limit (3.8) times a positive factor.

To show that $\mathscr{F}(k)$ has three roots in the interval $(1-n / 2,1)$, we show that

$$
\mathscr{F}(1-n / 2)<0, \quad \mathscr{F}(-1)>0, \quad \mathscr{F}(0)<0, \quad \mathscr{F}(1)>0
$$

First of all, we have $Q_{4}(-2)=Q_{4}(n-4)=0$, so we easily get

$$
k=0,1-n / 2 \quad \Longrightarrow \quad \mathscr{F}(k)=-\frac{2(k+1)^{4}}{n-4} \cdot 8(n-2)(n-4)<0 \text {. }
$$

Using our definitions (2.3) and (3.14), we can also verify that

$$
\mathscr{F}(-1)=\lim _{k \rightarrow-1} \frac{2(k+1)^{4}}{n-4} \cdot Q_{4}\left(\frac{n-4}{k+1}\right)=2(n-4)^{3}>0,
$$

while the fact that $\mathscr{F}(1)>0$ follows by the Taylor expansion

$$
\begin{aligned}
\mathscr{F}(1) & =2 n^{3}-8 n^{2}-256 n+512 \\
& =2(n-13)^{3}+70(n-13)^{2}+550(n-13)+226 .
\end{aligned}
$$

This proves (3.15), which implies that $\mathscr{F}$ has three roots in $(1-n / 2,1)$.

To see that the fourth root lies in $(n / 2-5, n / 2-4)$, we now note that

$$
\begin{aligned}
\mathscr{F}(n / 2-5) & =2 n^{4}-60 n^{3}+608 n^{2}-2336 n+2432 \\
& =2(n-13)^{4}+44(n-13)^{3}+296(n-13)^{2}+628(n-13)+118
\end{aligned}
$$

is positive for each $n \geq 13$, whereas

$$
\begin{aligned}
\mathscr{F}(n / 2-4) & =-n^{4}+18 n^{3}-124 n^{2}+416 n-608 \\
& =-(n-13)^{4}-34(n-13)^{3}-436(n-13)^{2}-2470(n-13)-5171
\end{aligned}
$$

is negative. In particular, the fourth root of $\mathscr{F}$ must lie in $(n / 2-5, n / 2-4)$.

Case 1. If $1 \leq k \leq n / 2-5$, then $\mathscr{F}(k)$ is positive by above.

Case 2. If $k \geq n / 2-4$, then $\mathscr{F}(k)$ is negative by above.

Case 3. If $k=(n-9) / 2$ and $n$ is odd, finally, then we can readily check that 


$$
\mathscr{F}(k)=\frac{n-1}{2} \cdot\left(n^{3}-33 n^{2}+312 n-892\right)
$$

is positive if and only if $n \geq 20$. In any case then, the result follows easily.

\section{References}

[1] Caffarelli, L., Gidas, B., Spruck, J.: Asymptotic symmetry and local behavior of semilinear elliptic equations with critical Sobolev growth. Commun. Pure Appl. Math. 42, 271-297 (1989)

[2] Chen, W., Li, C.: Classification of solutions of some nonlinear elliptic equations. Duke Math. J. 63, 615-622 (1991)

[3] Chen, W., Li, C., Ou, B.: Classification of solutions for an integral equation. Commun. Pure Appl. Math. 59, 330-343 (2006)

[4] Deng, Y., Li, Y., Liu, Y.: On the stability of the positive radial steady states for a semilinear Cauchy problem. Nonlinear Anal. 54, 291-318 (2003)

[5] Ding, W.-Y., Ni, W.-M.: On the elliptic equation $\Delta u+K u^{(n+2) /(n-2)}=0$ and related topics. Duke Math. J. 52, 485-506 (1985)

[6] Ferrero, A., Grunau, H.-C., Karageorgis, P.: Supercritical biharmonic equations with power-type nonlinearity. Ann. Mat. Pura Appl. 188, 171-185 (2009)

[7] Gazzola, F., Grunau, H.-C.: Radial entire solutions for supercritical biharmonic equations. Math. Ann. 334, 905-936 (2006)

[8] Gui, C., Ni, W.-M., Wang, X.: On the stability and instability of positive steady states of a semilinear heat equation in $\mathbb{R}^{n}$. Comm. Pure Appl. Math. 45, 1153-1181 (1992)

[9] Guo, Z., Wei, J.: Qualitative properties of entire radial solutions for a biharmonic equation with supercritical nonlinearity. Proc. Am. Math. Soc. 138, 3957-3964 (2010)

[10] Karageorgis, P.: Stability and intersection properties of solutions to the nonlinear biharmonic equation. Nonlinearity 22, 1653-1661 (2009)

[11] Li, Y.: Asymptotic behavior of positive solutions of equation $\Delta u+K(x) u^{p}=0$ in $\mathbb{R}^{n}$. J. Differ. Equ. 95, 304-330 (1992)

[12] Lin, C.-S.: A classification of solutions of a conformally invariant fourth order equation in $\mathbf{R}^{n}$. Comment. Math. Helv. 73, 206-231 (1998)

[13] Wang, X.: On the Cauchy problem for reaction-diffusion equations. Trans. Am. Math. Soc. 337, 549-590 (1993)

[14] Wei, J., Xu, X.: Classification of solutions of higher order conformally invariant equations. Math. Ann. 313, 207-228 (1999)

[15] Winkler, M.: Refined asymptotics for entire solutions of a biharmonic equation with a supercritical nonlinearity. Math. Ann. 348, 633-666 (2010) 
Paschalis Karageorgis

School of Mathematics

Trinity College

Dublin 2

Ireland

e-mail: pete@maths.tcd.ie

Received: 15 May 2011.

Accepted: 8 September 2011. 